\title{
ON OSCILLATIONS OF UNBOUNDED SOLUTIONS
}

\author{
I. GYÖRI, G. LADAS AND L. PAKULA
}

(Communicated by Kenneth L. Meyer)

ABSTRACT. Consider the differential equation with deviating arguments

$$
\text { (1) } \dot{y}(t)+p\left[y\left(t-\sigma_{1}\right)-y\left(t-\sigma_{2}\right)\right]=0
$$

where $p, \sigma_{1}$ and $\sigma_{2}$ are real numbers. We prove that every unbounded solution of (1) oscillates if and only if the characteristic equation

(2) $\lambda+p\left(e^{-\lambda \sigma_{1}}-e^{-\lambda \sigma_{2}}\right)=0$

has no positive roots and 0 is a simple root of (2).

\section{INTRODUCTION}

Consider the differential equation with deviating arguments

$$
\dot{y}(t)+p\left[y\left(t-\sigma_{1}\right)-y\left(t-\sigma_{2}\right)\right]=0
$$

where $p, \sigma_{1}$ and $\sigma_{2}$ are real numbers. The characteristic equation of (1) is

$$
F(\lambda) \equiv \lambda+p\left(e^{-\lambda \sigma_{1}}-e^{-\lambda \sigma_{2}}\right)=0 .
$$

Our aim in this paper is to prove the following result.

Theorem 1. The following statements are equivalent:

(a) Every unbounded solution of equation (1) oscillates.

(b) Equation (2) has no positive roots and 0 is a simple root of equation (2).

As is customary, a solution of (1) is called oscillatory if it has arbitrarily large zeros. Otherwise it is called nonoscillatory.

We note that any nonzero constant is a (bounded, nonoscillatory) solution of (1) no matter what the values of $p, \sigma_{1}$ and $\sigma_{2}$, and hence we do not expect an analogous equivalence statement for bounded solutions.

When $\sigma_{1}$ and $\sigma_{2}$ are both delays, or both advanced arguments, condition (b) is equivalent to a simple condition on the parameters $p, \sigma_{1}$ and $\sigma_{2}$. (See (4), (7) below.) When $\sigma_{1}$ and $\sigma_{2}$ are both delays an elementary proof is available

Received by the editors July 31, 1988 and, in revised form, December 15, 1988.

1980 Mathematics Subject Classification (1985 Revision). Primary 34K15.

The first author is on leave from the Computing Centre of the Szeged University of Medicine, 6720 Szeged, Hungary. 
and we present this first. If $\sigma_{1}$ and $\sigma_{2}$ are mixed arguments then there is no such simple condition on $p, \sigma_{1}$ and $\sigma_{2}$ equivalent to (b). When at least one of the arguments $\sigma_{1}$ or $\sigma_{2}$ is advanced, we first show that a positive solution of (1) has a Laplace transform and then show, by complex integration techniques, that such a solution cannot be unbounded.

\section{Proof of Theorem 1}

We first show $(a) \Rightarrow(b)$. Equation (2) cannot have a positive root $\lambda$ for otherwise, $e^{\lambda t}$ would be an unbounded and nonoscillatory solution of (1). Also, 0 must be simple root of (2). Otherwise, $F^{\prime}(0)=0$ and so $1+p\left(-\sigma_{1}+\sigma_{2}\right)=0$. In this case, (1) has the unbounded and nonoscillatory solution $y(t)=t$, which is a contradiction.

Next we show that $(b) \Rightarrow(a)$. If $p=0$ or if $\sigma_{1}=\sigma_{2}$, equation (1) has no unbounded solutions and so the proof is complete. Therefore we will assume that $p\left(\sigma_{1}-\sigma_{2}\right) \neq 0$.

Without loss of generality we will also assume that $\sigma_{1}>\sigma_{2}$.

Suppose, for the sake of contradiction, that (1) has an eventually positive and unbounded solution $y(t)$. As (1) is autonomous, we may (and will) assume that

$$
y(t)>0 \quad \text { for } t \geq \min \left\{0,-\sigma_{1}\right\} .
$$

Set

$$
z(t)=y(t)-p \int_{t-\sigma_{1}}^{t-\sigma_{2}} y(s) d s \quad \text { for } t \geq 0 .
$$

Then $\dot{z}(t)=0$ and so

$$
y(t)=C+p \int_{t-\sigma_{1}}^{t-\sigma_{2}} y(s) d s \quad \text { for } t \geq 0,
$$

where

$$
C=y(0)-p \int_{-\sigma_{1}}^{-\sigma_{2}} y(s) d s
$$

is some constant. As $y(t)$ is positive and unbounded, it follows from (3) that $p>0$.

We have the following two cases to consider.

Case 1. $\sigma_{2} \geq 0$ (also by hypothesis $\sigma_{1}>\sigma_{2}$ ).

As 0 is a simple root of equation (2) and because $F(\infty)=\infty$, it follows that $F^{\prime}(0)>0$, that is

$$
p\left(\sigma_{1}-\sigma_{2}\right)<1 .
$$

In view of the assumption that $y(t)$ is unbounded, it follows that there exists a sequence of points $\left\{t_{n}\right\}$ such that

$$
\lim _{n \rightarrow \infty} t_{n}=\infty, \quad \lim _{n \rightarrow \infty} y\left(t_{n}\right)=\infty \quad \text { and } \quad y\left(t_{n}\right)=\max _{s \leq t_{n}} y(s)
$$


for $n=1,2 \ldots$ Then $(3)$ yields,

$$
y\left(t_{n}\right)=C+p \int_{t_{n}-\sigma_{1}}^{t_{n}-\sigma_{2}} y(s) d s \leq C+p\left(\sigma_{1}-\sigma_{2}\right) y\left(t_{n}\right)
$$

or

$$
\left[1-p\left(\sigma_{1}-\sigma_{2}\right)\right] y\left(t_{n}\right) \leq C,
$$

which, in view of (4) and (5) leads to a contradiction as $n \rightarrow \infty$.

Case 2. $\sigma_{2}<0$ (also by hypothesis $\sigma_{1}>\sigma_{2}$ ).

The proof in this case will proceed by a series of lemmas. For the remainder of this proof one should recall that $y(t)$ has been assumed positive and unbounded, and we are seeking a contradiction.

Lemma 1. There exists a positive number $c$ such that $y(t)=O\left(e^{c t}\right)$.

Proof. We will first show that, for some $c$,

$$
\int_{0}^{\infty} e^{-c t} y(t) d t<\infty
$$

As 0 is a simple root of $(2)$ and because $F(\infty)=-\infty$, it follows that $F^{\prime}(0)<0$, that is,

$$
p\left(\sigma_{1}-\sigma_{2}\right)>1 \text {. }
$$

Set $y(t)=z(t)+k$ where $k=C /\left[1-p\left(\sigma_{1}-\sigma_{2}\right)\right]$ and $C$ is the constant in (3). Then (3) yields

$$
z(t)=p \int_{-\sigma_{1}}^{-\sigma_{2}} z(t+s) d s
$$

and

$$
z(t) \geq-K \text { where } K=|k| \text {. }
$$

Now fix an $\varepsilon>0$ with $-\sigma_{1}<\varepsilon<-\sigma_{2}$ and choose $c>0$ so that

$$
\gamma \equiv p \int_{\varepsilon}^{-\sigma_{2}} e^{c s} d s>1
$$

We will show that for this $c$, the function $y(t)$ satisfies (6). To this end set

$$
w(t)=\int_{0}^{t} e^{-c u} z(u) d u
$$

and observe that

$$
\begin{aligned}
w(t) & =\int_{0}^{t} p e^{-c u} \int_{-\sigma_{1}}^{-\sigma_{2}} z(u+s) d s d u \\
& =p \int_{-\sigma_{1}}^{-\sigma_{2}} \int_{0}^{t} e^{-c u} z(u+s) d u d s \\
& =p \int_{-\sigma_{1}}^{-\sigma_{2}} e^{c s} \int_{s}^{t+s} e^{-c v} z(v) d v d s
\end{aligned}
$$


That is,

$$
w(t)=p \int_{-\sigma_{1}}^{-\sigma_{2}} e^{c s} w(t+s) d s-C_{1}
$$

where

$$
C_{1}=p \int_{-\sigma_{1}}^{-\sigma_{2}} e^{c s} w(s) d s
$$

Now observe that by (9) and (11)

$$
w(t) \geq-K / c \quad \text { for } t \geq 0
$$

and so (12) yields,

$$
\begin{aligned}
w(t) & =p \int_{-\sigma_{1}}^{-\sigma_{2}} e^{c s}[w(t+s)+K / c] d s-\frac{K p}{c} \int_{-\sigma_{1}}^{-\sigma_{2}} e^{c s} d s-C_{1} \\
& \geq p \int_{\varepsilon}^{-\sigma_{2}} e^{c s} w(t+s) d s-C_{2}
\end{aligned}
$$

where $C_{2}=K p\left(e^{-\sigma_{2} c}-e^{-\sigma_{1} c}\right) / c^{2}-C_{1}$.

By the mean value theorem for integrals and with $\gamma$ as chosen in (10), it follows from (14) that given any $t_{0}>0$ there exists a $t_{1} \in\left(t_{0}+\varepsilon, t_{0}-\sigma_{2}\right)$ such that $w\left(t_{0}\right)+C_{2} \geq \gamma w\left(t_{1}\right)$. By induction there is a sequence $\left\{t_{n}\right\}$ such that, for $n=0,1, \ldots$,

$$
\begin{gathered}
t_{n}+\varepsilon<t_{n+1}<t_{n}-\sigma_{2} ; \\
w\left(t_{n+1}\right) \leq w\left(t_{n}\right) / \gamma+C_{2} / \gamma .
\end{gathered}
$$

Consider the difference equation

$$
x_{n+1}=x_{n} / \gamma+C_{2} / \gamma
$$

for $n=0,1, \ldots$, with $x_{0}=w\left(t_{0}\right)$. It follows by induction that

$$
w\left(t_{n}\right) \leq x_{n}
$$

Clearly

$$
\lim _{n \rightarrow \infty} x_{n}=C_{2} /(\gamma-1)
$$

and so from (16) and (13) we conclude that the sequence $\left\{w\left(t_{n}\right)\right\}$ is bounded above by a constant, say $M$. Then for $n=0,1, \ldots$ we have

$$
\begin{aligned}
\int_{0}^{t_{n}} e^{-c s} y(s) d s & =\int_{0}^{t_{n}} e^{-c s}[z(s)+k] d s \\
& =w\left(t_{n}\right)+k \int_{0}^{t_{n}} e^{-c s} d s \\
& \leq M+K / c .
\end{aligned}
$$

It follows that the Laplace transform

$$
Y(s)=\int_{0}^{\infty} e^{-s t} y(t) d t
$$


of the positive function $y(t)$ exists for $\operatorname{Re} s>c$. Now multiply (3) by $e^{-c t}$ and note that for $s$ in the interval of integration we have $e^{-c t} \leq e^{-c s} e^{-c \sigma_{2}}$. It now follows from the finiteness of integral (17) that $e^{-c t}(y(t)-C) \rightarrow 0$ which establishes the lemma.

Define $\phi(s)$ for complex $s$ by

$$
\phi(s)=y(0)-p e^{-s \sigma_{1}} \int_{-\sigma_{1}}^{0} e^{-s t} y(t) d t+p e^{-s \sigma_{2}} \int_{-\sigma_{2}}^{0} e^{-s t} y(t) d t .
$$

Taking the Laplace transform of both sides of (1) we get, for $\operatorname{Re} s>c$, that

$$
F(s) Y(s)=\phi(s) \text {. }
$$

Fix a real number $a>c$. By the inversion theorem for Laplace transforms (as in [4], p. 108) we have

$$
y(t)=\lim _{r \rightarrow \infty} \frac{1}{2 \pi i} \int_{a-i r}^{a+i r} e^{s t} \phi(s) / F(s) d s .
$$

For notational convenience we will abbreviate such integrals by

$$
y(t)=\int_{(a)} e^{s t} \phi(s) / F(s) d s .
$$

We will prove that

$$
y(t)=L(t)+R(t)
$$

where $R(t)$ is a constant plus a finite number of sinusoids, and $L(t) \rightarrow 0$ as $t \rightarrow \infty$. We use techniques similar to those of $\S \S 4.2-4$ and 12.15 of [1].

Lemma 2. For any real number $b$, the characteristic function $F(s)$ has only finitely many roots in the vertical strip $b \leq \operatorname{Re} s<a$.

Proof. From the definition of $F(s)$ it is easy to see that for large enough $r>0$ we have $|F(x \pm i r)|>r / 2$ for $b \leq x<a$. In particular, there is an $M>0$ so that if $b \leq \operatorname{Re} s<a$ and $F(s)=0$, then $|\operatorname{Im} s|<M$, i.e. all zeros in the vertical strip actually lie in a finite rectangle. By analyticity of $F$ there are then only finitely many zeros in the strip.

Fix $a>c$ where $c$ is as in Lemma 1. It follows from Lemma 2 that we can choose a number $b<0$ so that in the strip $b \leq \operatorname{Re} s<a, \phi(s) / F(s)$ has only finitely many poles. In particular we can assume that $F(s) \neq 0$ for $\operatorname{Re} s=b$. Let $R(t)$ be the sum of the residues of $e^{s t} \phi(s) / F(s)$ arising from the poles in $b \leq \operatorname{Re} s<a$ and let

$$
L(t)=\int_{(b)}-e^{s t} \phi(s) / F(s) d s .
$$

We fix a value of $t$ for now and verify (19). To this end, consider the rectangular contour $\gamma$ with vertices $a \pm i r, b \pm i r$. Denote the vertical segments by $\gamma_{1}$ and $\gamma_{3}$, and the horizontal segments by $\gamma_{2}$ and $\gamma_{4}$. Then we claim

$$
\lim _{r \rightarrow \infty} \int_{\gamma_{k}} e^{s t} \phi(s) / F(s) d s=0
$$


for $k=2,4$. Recall that $t$ is fixed here. It is easy to check that there is an $m>0$ such that $\left|e^{s t} \phi(s)\right|<m$ for $s=x \pm i r$ with $b \leq x \leq a$ and any $r$. By the argument of Lemma 2 , we see that $|1 / F(x \pm i r)|<2 / r$ for all $b \leq x \leq a$ and all large enough $r$. Thus for $k=2,4$ we have

$$
\int_{\gamma_{k}} e^{s t} \phi(s) / F(s) d s=O(1 / r)
$$

as $r \rightarrow \infty$ which establishes the claim.

Thus (19) follows from the residue theorem and the inversion theorem. Next we show that $L(t) \rightarrow 0$ as $t \rightarrow \infty$. Let us write $\phi(s)=\phi_{0}(s)+\phi_{1}(s)+\phi_{2}(s)$ corresponding to the three terms on the right in (18). We show

$$
\int_{(b)} e^{s t} \phi_{k}(s) / F(s) d s \rightarrow 0
$$

as $t \rightarrow \infty$, for $k=0,1$ and 2 .

First consider $k=1$. Then $\phi_{0}(s)$ is the constant $y(0)$ which we take to be 1 for convenience. Define $f(w)=F(b+i w)$ for real $w$ and let

$$
g(t)=\int_{(b)} e^{s t} / F(s) d s=\lim _{r \rightarrow \infty} e^{b t} \int_{-r}^{r} e^{i w t} / f(w) d w
$$

Also, define

$$
T(w)= \begin{cases}1 / i w, & \text { if }|w| \geq 1 \\ 0, & \text { otherwise. }\end{cases}
$$

From our assumption on $b, f(w) \neq 0$ for all $w$. We see from the explicit form of $f(w)$ that $|1 / f(w)-T(w)|=O\left(1 / w^{2}\right)$ as $w \rightarrow \infty$ and we thus conclude that $1 / f(w)-T(w)$ is absolutely integrable so that

$$
\lim _{r \rightarrow \infty} \int_{-r}^{r} e^{i w t}[1 / f(w)-T(w)] d w=\int_{-\infty}^{\infty} e^{i w t}[1 / f(w)-T(w)] d w .
$$

This last integral is bounded in $t$. On the other hand, explicit contour integration shows that as $t \rightarrow \infty$,

$$
\lim _{r \rightarrow \infty} \int_{-r}^{r} e^{i w t} T(w) d w \sim \frac{1}{t} .
$$

Now combining (23) and (24), and using $b<0$, we see from (22) that $g(t) \rightarrow 0$ as $t \rightarrow \infty$.

Next, consider $\phi_{k}$ with $k=1$ or 2 . We take $k=1$ to be specific. Again, let $f(w)=F(b+i w)$ for real $w$, but let

$$
g(t)=\int_{(b)} e^{s t} \phi_{1}(s) / F(s) d s=\lim _{r \rightarrow \infty} e^{b t} \int_{-r}^{r} e^{i w t} \Phi_{1}(w) / f(w) d w
$$

where $\Phi_{1}(w)=\phi_{1}(b+i w)$. A change of variable in the definition of $\phi_{1}(s)$ gives

$$
\Phi_{1}(w)=\int_{-2 \sigma_{2}}^{-\sigma_{1}} e^{i w \tau} e^{b \tau} y\left(\tau+\sigma_{1}\right) d \tau
$$


We see from this that $\Phi_{1}$ is the Fourier transform of a bounded function of $\tau$ with compact support, that function thus being in both $L^{1}(d \tau)$ and $L^{2}(d \tau)$. But the ordinary Fourier transform of such a function is in $L^{2}(d w)$ ([3], p. 187). Since $f(w) \neq 0$ and $|1 / f(w)|=O(1 /|w|)$ as $w \rightarrow \infty$, we conclude that $1 / f(w)$ is in $L^{2}(d w)$. As the product of two functions in $L^{2}(d w)$ is in $L^{1}(d w)$ it follows that $\Phi_{1}(w) / f(w) \in L^{1}$. Hence

$$
g(t)=e^{b t} \int_{-\infty}^{\infty} e^{i w t} \Phi_{1}(w) / f(w) d w
$$

and as in the previous case we can show $g(t) \rightarrow 0$ as $t \rightarrow \infty$.

We have thus shown that $L(t) \rightarrow 0$ in (19). Let the distinct zeros of $F(s)$ in the strip $b \leq \operatorname{Re} s<a$ be denoted by $s_{0}, s_{1}, \ldots, s_{2 n}$, with $s_{0}=0$. Note that the other zeros are in conjugate pairs. Then $R(t)$ has the form

$$
\sum_{k} P_{k}(t) e^{s_{k} t}
$$

where $P_{k}(t)$ is a polynomial (with complex coefficients) of degree less than the multiplicity of the zero $s_{k}$ [1, p. 108]. Since 0 is a simple root of (2) by hypothesis, $\operatorname{deg} P_{0}=0$, so the term corresponding to $k=0$ is a constant.

If $n=0$ then $R(t)$ is constant and $y(t)$ is bounded, a contradiction. Otherwise, the real part of $R(t)$ can be written as

$$
\text { const }+\sum_{k=1}^{n} e^{r_{k} t}\left(p_{k}(t) \cos \theta_{k} t+q_{k}(t) \sin \theta_{k} t\right)
$$

where $p_{k}(t)$ and $q_{k}(t)$ are polynomials with real coefficients and $r_{k} \geq 0$. We need the following lemma. A simple proof is included for the reader's convenience.

Lemma 3. For any sum

$$
f(t)=\sum_{k=1}^{n} a_{k} \cos \theta_{k} t+b_{k} \sin \theta_{k} t
$$

which is not identically zero and for which all $\theta_{k} \neq 0$, there exists $\varepsilon>0$ such that $\lim \sup _{t \rightarrow \infty} f(t)>\varepsilon$ while $\liminf _{t \rightarrow \infty} f(t)<-\varepsilon$.

Proof. Since $f(t)$ is an almost-periodic function, the result follows easily from the (Bohr) definition of almost periodicity (see e.g. [2]) if we can show that $f(t)$ takes on both positive and negative values. Observe that any $f(t)$ as in the lemma has mean value

$$
M(f)=\lim _{T \rightarrow \infty} \frac{1}{T} \int_{0}^{T} f(t) d t
$$

zero. Suppose, say, $f(t) \geq 0$ for all $t$. Then $f(t)$ would have an indefinite integral $F(t)$ of the same form as $f$, with $M(F)=0$, and with $F(t)$ nondecreasing and bounded. Then $\lim _{t \rightarrow \infty} F(t)=L$ for some $L$, so $M(F)=L=0$. But, 
assuming as we may, all $\theta_{k}$ distinct and positive, $M\left(F^{2}\right)=\sum\left(a_{k}^{2}+b_{k}^{2}\right) / 2 \theta_{k}^{2}>0$ by our assumptions on $f$, so $0<M\left(F^{2}\right)=L^{2}$; a contradiction.

In (28), let $r_{M}$ be the largest of the $r_{k}$ 's and let $m$ be the highest degree among the $p_{k}$ 's and $q_{k}$ 's with $r_{k}=r_{M}$. Letting $\alpha_{k}, \beta_{k}$ be the coefficients of $t^{m}$ in $p_{k}, q_{k}$, set

$$
R_{1}(t)=e^{r_{M} t} t^{m} \sum \alpha_{k} \cos \theta_{k} t+\beta_{k} \sin \theta_{k}
$$

where the sum is over those $k$ for which $r_{k}=r_{M}$. If $r_{M}>0$ or if $r_{M}=0$ but $m>0$ we could find, using Lemma 3 , a sequence $t_{n} \rightarrow \infty$ for which $\liminf _{n \rightarrow \infty} R_{1}\left(t_{n}\right)=-\infty$ while $R\left(t_{n}\right) \sim R_{1}\left(t_{n}\right)$ as $n \rightarrow \infty$. In view of (19), this contradicts the positivity of $y(t)$. Thus $r_{M}=0, m=0$, and $R(t)$ is bounded; hence $y(t)$ is bounded. This contradiction completes the proof of the theorem.

\section{REFERENCES}

1. R. Bellman and K. Cooke, Differential-difference equations, Academic Press, New York, 1963.

2. C. Corduneanu, Almost-periodic functions, Interscience Publishers, New York, 1968.

3. W. Rudin, Real and complex analysis, McGraw-Hill, New York, 1966.

4. D. V. Widder, An introduction to transform theory, Academic Press, New York, 1971. 02881

Depa.rtment of Mathematics, University of Rhode Island, Kingston, Rhode Island 